\title{
Perforación de colon sigmoides secundaria a endometriosis. Reporte de un caso
}

\author{
Perforation of the sigmoid colon secondary to endometriosis. Case report
}

Ernesto A. Dzib-Calan ${ }^{*}$, Jorge I. Morales-Pérez', Juan C. Aranda-Puebla², Álex Simón-Mendoza², Gerardo Rodarte-Cajica ${ }^{2}$ y Gustavo Leal-Mérida ${ }^{3}$

${ }^{1}$ Escuela de Posgrado de Sanidad Naval; ${ }^{2}$ Servicio de Cirugía general; ${ }^{3}$ Áreas quirúrgicas. Hospital General Naval de Alta Especialidad, Ciudad de México, México

\section{Resumen}

Antecedentes: La endometriosis es la presencia de glándulas endometriales o estroma viable fuera de la cavidad uterina, que afecta aproximadamente al 2-10\% de las mujeres en edad reproductiva1. Es común la afección de estructuras pélvicas, incluyendo el intestino. La perforación del colon por endometriosis es muy rara y representa una urgencia quirúrgica. Caso clínico: Mujer de 28 años con cuadro de dolor abdominal en fosa iliaca derecha y hueco pélvico, fiebre y náuseas. Se realiza laparotomía exploradora con hallazgo de perforación de colon sigmoides, que requiere resección de la lesión y colostomía terminal, encontrando como diagnóstico definitivo endometriosis. Conclusión: La perforación de intestino o de colon es una complicación poco frecuente, pero de gravedad, que debemos tener siempre presente como sospecha ante un cuadro de abdomen agudo en una paciente en edad fértil y con antecedentes de haber presentado sintomatología gastrointestinal intermitente.

PALABRAS CLAVE: Endometriosis. Intestino. Colon. Perforación.

\begin{abstract}
Background: Endometriosis is the presence of endometrial glands or viable stroma outside the uterine cavity, which affects approximately $2-10 \%$ of women of reproductive age 1. Pelvic structures, including the bowel, are commonly affected. Perforation of the colon by endometriosis is very rare and represents a surgical emergency. Clinical case: A 28-year-old female patient with abdominal pain in the right iliac fossa and pelvic cavity, fever and nausea, exploratory laparotomy is performed with the discovery of sigmoid perforation of the colon, requiring resection of the lesion and terminal colostomy, finding as definitive diagnosis endometriosis. Conclusion: Bowel or colon perforation is a rare but serious complication, which should always be kept in mind as a suspicion of acute abdomen in a female patient of reproductive age and with a history of intermittent gastrointestinal symptoms.
\end{abstract}

KEY WORDS: Endometriosis. Intestine. Colon. Perforation.

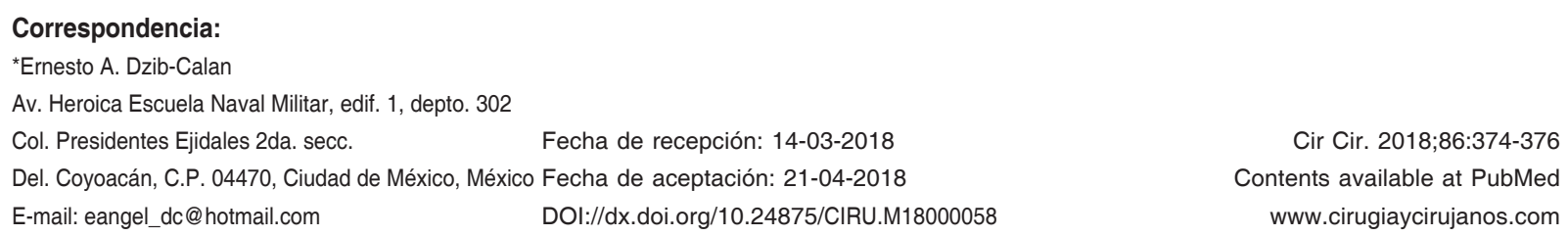




\section{Introducción}

La endometriosis es la presencia de glándulas endometriales o de estroma viable fuera de la cavidad uterina, que afecta aproximadamente al $2-10 \%$ de las mujeres en edad reproductiva'. Es común la afección de estructuras pélvicas, incluyendo el intestino.

La prevalencia de endometriosis intestinal se encuentra entre el 5.3 y el $12 \%$. El recto y el colon sigmoides son los más afectados, siendo el íleon el más raramente afectado ${ }^{2}$. La edad promedio de diagnóstico es de 34-40 años ${ }^{3}$. La perforación del colon por endometriosis es muy rara y en general las pacientes se presentan asintomáticas o con una masa dolorosa pélvica en la fosa iliaca izquierda. La falta de signos y síntomas patognomónicos hace de la endometriosis intestinal una enfermedad de difícil sospecha y raro diagnóstico preoperatorio. Los diagnósticos diferenciales incluyen síndrome del intestino irritable, enfermedades infeccionas, isquemia mesentérica, síndrome de Crohn y neoplasia ${ }^{4}$.

\section{Caso clínico}

Mujer de 28 años de edad con antecedentes quirúrgicos de cesárea hace 2 años por sufrimiento fetal agudo, fecha de última menstruacion (FUM) 15 días antes de su ingreso, sin enfermedades crónicas degenerativas, que acude al servicio de urgencias por dolor abdominal de 7 días de evolución localizado en el hipogastrio y la fosa iliaca derecha, de intensidad $7 / 10$ en la escala análoga del dolor, tipo cólico, sin otra sintomatología acompañante, manejada de manera externa con butilhoscina, metamizol, clonixinato de lisina, cisaprida, omeprazol y ciprofloxacino, sin mejoría en 24 horas. Acude nuevamente con aumento del dolor de 12 horas de evolución, acompañado de náuseas sin llegar al vómito, anorexia, fiebre no cuantificada y disminución del dolor con posición gatillo; niega sintomatología genitourinaria o genital; última evacuación 16 horas antes, de escasa cantidad con características normales.

En la exploración física se encuentra: presión arterial 110/60 mmHg, frecuencia cardiaca 112 latidos por minuto, frecuencia respiratoria 20 respiraciones por minuto, temperatura $38.2{ }^{\circ} \mathrm{C}$, neurológicamente íntegra, sin alteraciones cardiopulmonares, abdomen globoso a expensas de panículo adiposo, cicatriz tipo Pfannenstiel, blando, depresible; McBurney, Von Blumberg, psoas, talopercusión y obturador positivos; Murphy, puntos ureterales, pancreáticos y Giordanos

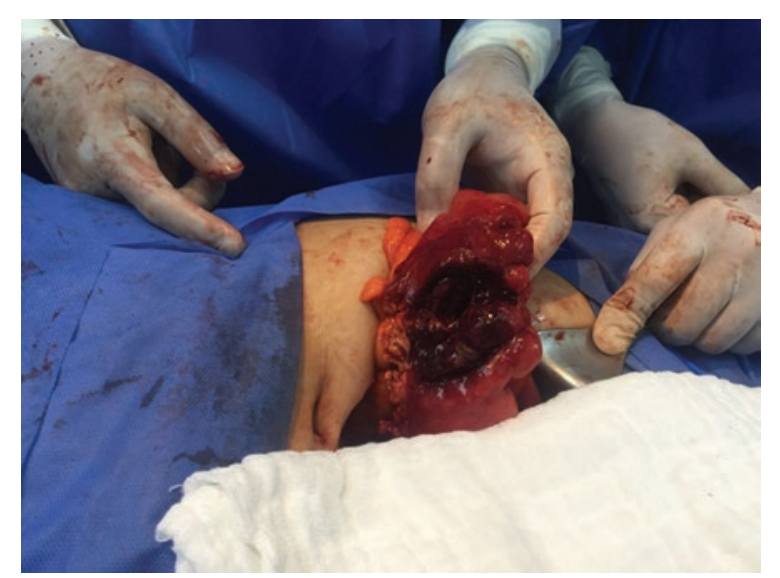

Figura 1. Perforación de sigmoides.

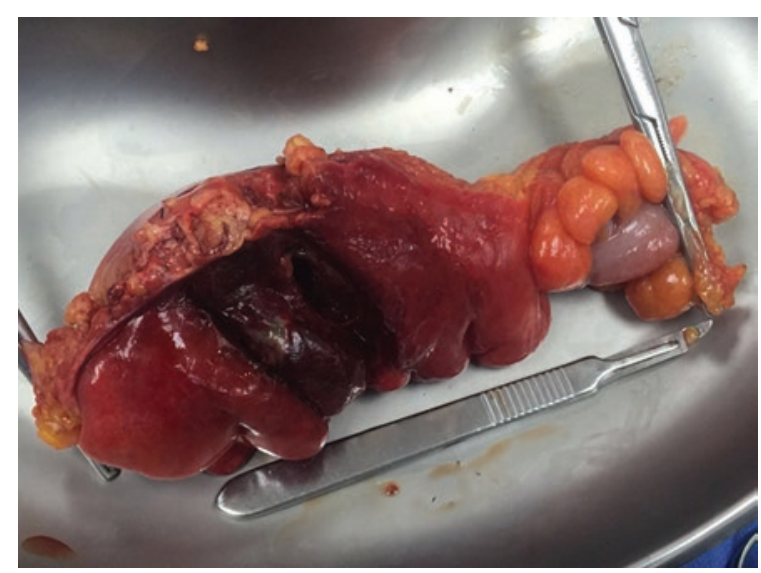

Figura 2. SegXmento de sigmoides.

negativos; extremidades íntegras, sin compromiso neurovascular.

Biometría hemática: leucocitos $16.8 \times 10^{3} / /$, neutrófilos $78.2 \%$, hemoglobina $9.1 \mathrm{mg} / \mathrm{dl}$, plaquetas 589,000 , prueba de embarazo negativa.

Se realiza laparotomía exploradora bajo sospecha de apendicitis, con los siguientes hallazgos: apéndice cecal de características normales, absceso en región de colon sigmoides de aproximadamente $100 \mathrm{ml}$, coIon sigmoides con aumento de volumen de $10 \times$ $10 \mathrm{~cm}$, con tejido adiposo de color café rojizo, con natas de fibrina, y dos lesiones de pérdida de continuidad en su superficie de disposición transversal de 3.5 y $5 \mathrm{~cm}$ (Figs. 1 y 2). Se realiza sigmoidectomía, cierre de muñón distal en bolsa de Hartman y colostomía terminal.

Reporte de patología: serosa y tejido adiposo de sigmoides con presencia de glándulas endometriales tubulares sin atipias en su epitelio, rodeadas por estroma endometrial fusocelular sin atipias; receptor de estrógenos positivo nuclear en epitelio de glándulas 
endometriales; citoqueratina 7 positiva en epitelio de glándulas endometriales; CD 10 positivo en estroma endometrial; y CD 34 positivo en endotelio vascular, con lo cual se ratifica como focos de endometriosis.

\section{Discusión}

La endometriosis es una enfermedad considerada benigna, pero puede presentar un comportamiento agresivo y ocasionar complicaciones graves. La endometriosis intestinal plantea un reto diagnóstico importante para cirujanos, ginecólogos y gastroenterólogos durante su práctica.

Se afectan comúnmente la serosay las capas musculares del intestino, mientras que la participación transmural en la mucosa es rara. La participación de la mucosa es la forma más grave de endometriosis intestinal. Las localizaciones más frecuentes son el recto y el colon sigmoides (73\%), mientras que la participación del intestino delgado (2-16\%), el apéndice (3-18\%), el ciego (2-5\%) y el íleon $(4,1 \%)$ es excepcional ${ }^{2,5}$.

Setubal y Sidiropoulou ${ }^{6}$ reportaron tres casos de endometriosis, todos en pacientes embarazadas, dos de las cuales se conocían con historia de endometriosis y una sin antecedentes de importancia. Todas debutaron con cuadros agudos de dolor abdominal en la región pélvica y requirieron laparotomía exploradora. En los tres casos se presentó afectación de colon sigmoides y se realizó la resección del segmento afectado.

Galazis, et al. ${ }^{5}$ presentan el caso de una paciente no embarazada con un cuadro de distensión, náuseas y vómitos de 1 mes de evolución, que acude por dolor súbito en la fosa iliaca izquierda con irradiación a la región suprapúbica, y antecedente de laparoscopia por endometriosis. Se pasa a laparotomía exploradora y se encuentra perforación de sigmoides, por lo que se realizan hemicolectomía izquierda y colostomía terminal.

Garg y Bagul 7 presentan el caso de una paciente con historial de 10 días de dolor cólico en el flanco y la fosa iliaca izquierdos, con antecedente de endometriosis. Pasa a laparotomía exploradora y se encuentra masa endometriósica en el ovario y la salpinge izquierdos, con involucro y perforación del sigmoides. Se realizan hemicolectomía izquierda y colostomía terminal, así como resección de la masa endometriósica y del anexo izquierdo.

La paciente de nuestro caso, a diferencia de lo mayormente reportado, no contaba con antecedentes de endometriosis, lo que hizo más difícil la sospecha. El diagnóstico puede resultar difícil, pues la mayoría de las veces no hay antecedentes sugestivos de endometriosis, los signos y síntomas no son específicos, y las pacientes a menudo acuden con un cuadro de abdomen agudo que requiere laparotomía exploratoria.

\section{Conclusión}

Esta es una complicación poco frecuente, pero de gravedad. La perforación se presenta principalmente asociada al embarazo, al final de este y en el puerperio, y son muy pocos los casos reportados sin asociación al embarazo. El manejo debe ser multidisciplinario. Siempre hay que tener presente esta enfermedad como sospecha ante un cuadro de abdomen agudo en una mujer en edad fértil y con antecedentes de sintomatología gastrointestinal intermitente. El antecedente de endometriosis o la coexistencia de síntomas ginecológicos deben aumentar el índice de sospecha, y la laparoscopia antes de la laparotomía formal debería considerarse ante la evidencia de perforación intestinal.

\section{Bibliografía}

1. Costa A, Sartini A. Deep endometriosis induced spontaneous colon rectal perforation in pregnancy: laparoscopy is advanced tool to confirm diagnosis. Case Rep Obstet Gynecol. 2014;2014:907150.

2. Albareda J, Albi MV, Sosa G, Cano A, Macello ME, Albi Martin B. Puerperal ileal perforation secondary to endometriosis: case report and literature review. Taiwan J Obstet Gynecol. 2016;55:121-4.

3. Dimoulios P, Koutroubakis IE, Tzardi M, Antoniou P, Matalliotakis IM, Kouroumalis EA. A case of sigmoid endometriosis difficult to differentiate from colon cancer. BMC Gastroenterol. 2003;3:18.

4. Tong YL, Chen Y, Zhu SY. lleocecal endometriosis and a diagnosis dilemma: a case report and literature review. World J Gastroenterol. 2013;19:3707e10.

5. Galazis N, Arul D, Wilson J, Pisal N. Bowel endometriosis. BMJ Case Rep. 2014. pii: bcr2013202140. doi: 10.1136/bcr-2013-202140.

6. Setubal A, Sidiropoulou Z. Bowel complications of deep endometriosis during pregnancy or in vitro fertilization. Fertil Steril. 2014;101:442-6.

7. Garg NK, Bagul NB. Intestinal endometriosis - a rare cause of colonic perforation. World J Gastroenterol. 2009;15:612-4. 